\title{
Spontaneous pneumomediastinum
}

\author{
Emmet E. McGrath MB PhD, Zoe Blades MB, Chris Barber MD
}

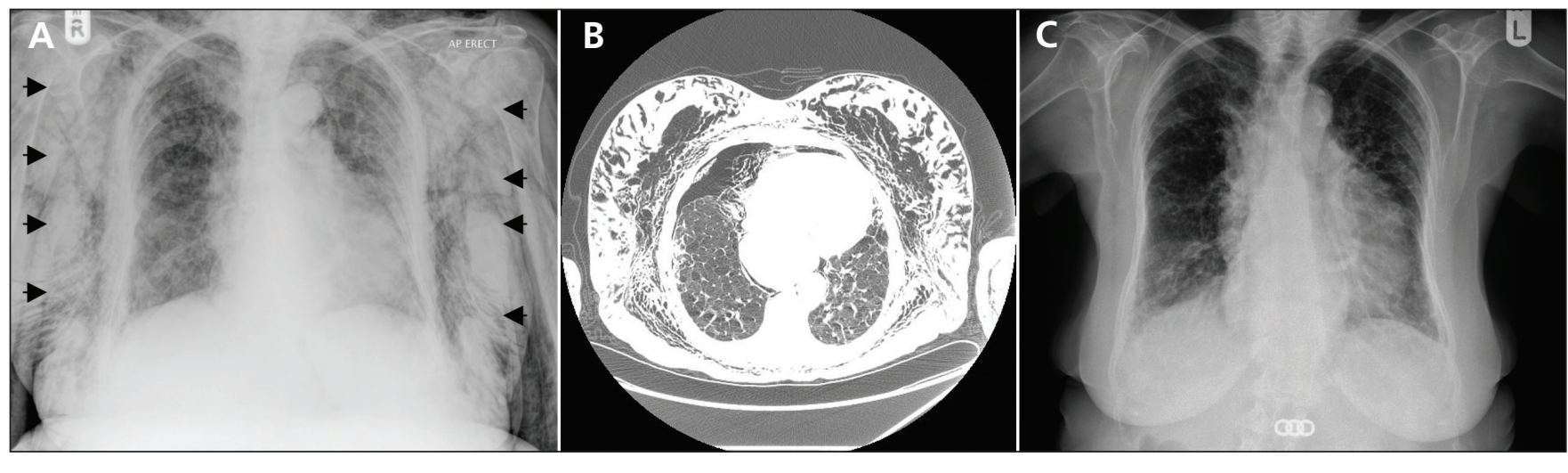

Figure 1: A: A radiograph of the patient's chest showing widespread opacification of the soft tissues of the thoracic cavity (arrows). B: A computed tomogram of the patient's thorax taken at presentation showing severe emphysema, as well as a right-sided pneumothorax, pneumomediastinum, and widespread subcutaneous emphysema. C: A radiograph of the patient's chest taken 5 days after presentation showing resolution of the subcutaneous emphysema.

A n 82-year-old woman with chronic obstructive pulmonary disease presented to the emergency department with atraumatic swelling of the chest and neck. On examination, she had soft tissue crepitus in the area of the swelling. Her breathing was slightly laboured, and the result of the oxygen saturation test was unremarkable. Spontaneous pneumomediastinum and subcutaneous emphysema were confirmed by imaging (Figure 1A, 1B). We suspected that an intense coughing episode that had occurred 24 hours before presentation caused the condition. She was given oxygen but declined to have a chest drain inserted or to have other invasive investigations. Her condition improved quickly, and a radiograph of her chest taken a few days later showed that the initial findings had resolved (Figure 1C).

Spontaneous pneumomediastinum tends to occur after intense episodes of increased intrathoracic pressure (e.g., coughing, straining, vomiting, strenuous crying), especially in people with asthma or chronic obstructive pulmonary disease. Alveoli that have ruptured release air, which travels to the lung hilum and then to the mediastinum. From there, the air often escapes to the pleura or subcutaneous tissue. Pneumo-

From the Department of Respiratory Medicine, Royal Hallamshire Hospital, Sheffield, UK

Cite as CMAJ 2009. DOI:10.1503/cmaj.081661 thorax, cardiac tamponade, tension pneumomediastinum and airway obstruction may also occur.

Most patients with spontaneous pneumomediastinum present with chest pain and up to half experience dyspnea. ${ }^{1,2}$ Subcutaneous emphysema is common, leading to crepitus on palpation, and systolic crepitation and reduced heart sounds (Hamman sign) with precordial auscultation. Spontaneous pneumomediastinum is most commonly seen in men in their 20s.

Computed tomography can be used to confirm the diagnosis and to exclude other causes of air leakage, such as Boerhaave syndrome (esophageal rupture following vomiting). Treatment of spontaneous pneumomediastinum is usually conservative, but insertion of a chest tube may help patients with associated pneumothorax or tracheal compression. The condition usually resolves spontaneously within 7 days, ${ }^{3}$ and recurrence is extremely low.

This article has been peer reviewed.

Competing interests: None declared.

\section{REFERENCES}

1. Caceres M, Ali SZ, Braud R, et al. Spontaneous pneumomediastinum: a comparative study and review of the literature. Ann Thorac Surg 2008;86:962-6.

2. Macia I, Moya J, Ramos R, et al. Spontaneous pneumomediastinum: 41 cases. Eur J Cardiothorac Surg 2007;31:1110-4.

3. Takada K, Matsumoto S, Hiramatsu T, et al. Management of spontaneous pneumomediastinum based on clinical experience of 25 cases. Respir Med 2008;102:1329-34. 